\title{
Superluminality and Entanglement in an Electromagnetic Quantum-Relativistic Theory
}

\author{
Massimo Auci ${ }^{1,2}$ \\ ${ }^{1}$ Maths and Physics Department, Statal European International School “Altiero Spinelli”, Torino, Italy \\ ${ }^{2}$ Space Science Department, Odisseo-Space, Milano, Italy \\ Email: massimo.auci@gmail.com
}

How to cite this paper: Auci, M. (2018) Superluminality and Entanglement in an Electromagnetic Quantum-Relativistic Theory. Journal of Modern Physics, 9, 2206-2222. https://doi.org/10.4236/jmp.2018.912139

Received: September 13, 2018

Accepted: October 15, 2018

Published: October 18, 2018

Copyright $\odot 2018$ by author and Scientific Research Publishing Inc. This work is licensed under the Creative Commons Attribution International License (CC BY 4.0).

http://creativecommons.org/licenses/by/4.0/

\begin{abstract}
An electromagnetic theory that links quantum and relativistic phenomena in a single context is built. Wave-particle duality is the experimental proof of their common origin. In this context, Quantum Mechanics and Special Relativity are two compatible synergistic theories. The developed theory shows the existence of superluminal effects that suggest an explanation to the entanglement between pairs of particles and photons.
\end{abstract}

\section{Keywords}

Electromagnetic Interactions, Electrodynamics, Dipoles, Quantum-Relativistic Effects, Photons Exchange, Quanta, Superluminality, Entanglements

\section{Introduction}

The Bridge Theory (BT) is a quantised electromagnetic approach to electrodynamics [1]. The theory is based on the proof of the conjecture [2] of the physical meaning of the transversal component of the Poynting vector in the dynamics of the interactions among pairs of charged particles. Starting from the BT, will extend in a self-consistent way the theory to Special Relativity, proving as the wave-particle duality principle is the experimental evidence that Quantum Mechanics and Special Relativity derive from a common quantum-relativistic phenomenology [3]. In this context an explanation to superluminality and to quantum entanglement is proposed.

The theory is based on the lack of spherical symmetry in the electromagnetic emission of a dipole, which is localising in its neighbourhood an amount of energy and momentum at charge of the transversal component of the Poynting 
vector of the Dipolar Electromagnetic Source (DEMS). The theoretical results concerning the localised energy and moment are in agreement with those of a photon. The BT provides two incertitude principles, one for observers inside the wave front of the DEMS, and one for observers placed outside the wave front in agreement with the Heisenberg's one. Also the theoretical and numerical estimations of the fine structure constant and consequently of the Planck constant, provided self-consistently from the theory, give results in agreement with the experimental one [4] [5].

To develop the outline of the theory it is assumed that the elementary particles in the lab system are already created in pair, each with a rest energy, whose origin and value are not now relevant for our purposes.

Following the BT, the total energy and momentum of each interacting pair settle the wavelength of the corresponding DEMS, which is equivalent to an exchanged photon with an energy and a momentum defined by particles dynamics. Three fundamental statements based on the actual theory are:

1) When a charged particle is moving in a medium, it produces with all the anti-particles with which it is in causal contact a spacetime distribution of DEMS;

2) The value of action characterising the production of a DEMS is the Planck constant which is depending by the internal structure of the electromagnetic field;

3) The DEMS is an electromagnetic source localising an energy and a momentum identical to those of the photon exchanged in the interaction between the pairs of particles forming the dipole; its wavelength is the synthesis of all the information about the dynamical state of the interacting particles.

\section{Spacetime Distribution of DEMS, Real Waves-Packet and Virtual Photons}

In order to observe a charged particle, according to the statement (I) we need that it interacts with at least another anti-particle producing a DEMS with a specific wavelength, this is equivalent to perform a measure of energy and momentum on the particle. When a charged particle crosses a space filled of matter feels the electromagnetic fields originated by the other particles and it interacts with them producing a distribution of DEMS. The multi-interaction shares the energy and momentum carried by the particle in motion with the distribution of DEMS, each with a different wavelength, the overlap of the waves originates a localised electromagnetic waves-packet which motion describes the incoming particle in spacetime.

The process producing a DEMS can be broken up into two phases:

- the approach or Alpha phase (A-phase), the interaction localises the energy and momentum of a photon inside the source zone;

- the removal or Omega phase ( $\Omega$-phase), the initial source zone is destroyed and the products emerging go away from one another continuing to interact. 
The wave emission occurs during both phases, only during the A-phase a part of the mechanical energy and momentum of the incoming charged particles is localised inside the source zone supplying the energy and momentum of the exchanged photon between the two interacting particles. Since in BT the Planck's action is weakly varying with external constrains, only for free interactions among pairs of charges its value is perfectly equal to the that of the Planck's constant, so the presence of phenomena strictly depending on Planck's action can be considered the evidence of the formation in spacetime of DEMS. If a DEMS is existing, a photon and an electromagnetic wave associated respectively to the transversal and radial components of the Poynting vector are coexisting in the same phenomenon. Following this idea, for a particle crossing a medium a distribution of DEMS in space realises a net of direct (photon exchange) and indirect electromagnetic connections among different observers, producing a phenomenology which agrees both with the quantum and waves behaviours.

\section{Formulation of a Quantum-Relativistic Principle}

When a DEMS is produced, an observer placed in the laboratory $S$ sees a fraction of the energy and momentum carried by the interacting particles exchanged between the particle as a photon, the residual amounts of energy and momentum provide respectively the kinetic energy and the momentum of the centre of mass of the source in motion with respect to $S$.

In order to characterise dynamically the interacting particles with respect to an observer $S$, we assign the initial energies and momenta $\left(E_{1}, \boldsymbol{P}_{1}\right)$ and $\left(E_{2}, \boldsymbol{P}_{2}\right)$. Before the beginning of the A-phase, the available total energy and momentum of the particles are given by

$$
\left\{\begin{array}{l}
E=E_{1}+E_{2} \\
\boldsymbol{P}=\boldsymbol{P}_{1}+\boldsymbol{P}_{2}
\end{array}\right.
$$

Considering the interaction occurring with the energy and momentum (1) with respect to the observer $S$; let $E_{\Gamma}$ and $\boldsymbol{P}_{\Gamma}$ be respectively energy and momentum of the exchanged photon, the conservation laws applied to the interacting particles and to the DEMS produced require:

$$
\left\{\begin{array}{l}
E_{\Gamma}=P_{\Gamma} c \\
\Delta_{0}=E-E_{\Gamma} \\
\frac{\Delta}{c}=\boldsymbol{P}-\boldsymbol{P}_{\Gamma}
\end{array}\right.
$$

where $\Delta_{0}$ and $\Delta / c$ are respectively energy and momentum not involved in the DEMS formation, associated to the transverse projection of the motion of the source when it is observed along the sight line of the observer placed in the lab frame $S$.

To solve the Equation (2) we consider the length of the residual momentum not observed along the sight line

$$
\Delta=\left\|\boldsymbol{P}-\boldsymbol{P}_{\Gamma}\right\| c=\sqrt{P^{2} c^{2}+P_{\Gamma}^{2} c^{2}-2 P P_{\Gamma} c^{2} \cos \theta}
$$


Considering the difference between the squared of the momentum (3) and the squared of the energy non-involved in the DEMS formation as defined in Equation (2), we obtain

$$
\Delta^{2}-\Delta_{0}^{2}=2 E_{\Gamma}(E-P c \cos \theta)-\left(E^{2}-P^{2} c^{2}\right)
$$

where $\theta$ is the angle between the total momentum $\boldsymbol{P}$ and the momentum $\boldsymbol{P}_{\Gamma}$ associated to the photon emitted along the direction of the sight line of the observer $S$.

In order to define the value of the left side term of the Equation (4), we consider initially the case of an observer $S_{0}$ in the centre of mass (c.m.) of the DEMS during a head-to-head interaction. The energy and the momentum (1) of the particles are completely involved in the DEMS formation, so the residual energy $\Delta_{0}$ and the transversal momentum $\Delta / c$ for this observer must be both equal to zero. If during the production of the DEMS a migrating inertial observer moves along its sight line transiting trough the c.m. of the source, the total energy and momentum observed cannot change, so the changing in energy and momentum of the source emission is balanced by the energy and momentum of the relative motion between c.m. of the source and observer in such a way that the value of the left side term of the Equation (4) continue to be zero for all the inertial observers even if the residual energy and transversal momentum are not zero as in c.m. Therefore, for each inertial observer embedded in the electromagnetic field of a DEMS, the squared difference at left side of the Equation (4) equal to the rest energy of the interacting particles not involved in the DEMS production is zero:

$$
\Delta^{2}-\Delta_{0}^{2}=0,
$$

using (5), the (4) can be solved with respect $E_{\Gamma}$ obtaining:

$$
E_{\Gamma}=\frac{E^{2}-P^{2} c^{2}}{2(E-P c \cos \theta)}
$$

able to measure along the direction defined by the observation angle $\theta$ the energy $E_{\Gamma}$ emitted by a DEMS in relative motion with respect the observer $S$.

The Equation (6) is able to manage energy and momentum of the DEMS observed in the frame $S$, its validity is general and introduces a fundamental principle involving the energy-momentum invariance for whatever system observer-source, independently from their relative inertial motion. Using the Equation (5) we propose the invariant

$$
\left(E-E_{\Gamma}\right)^{2}-\left(P-P_{\Gamma}\right)^{2} c^{2}=0
$$

as a Quantum-Relativistic Principle (QRP).

\section{The QRP Applied to a Single Moving Particle}

By considering an observer $S$ placed in a frame associated to one of two interacting particles of a pair, for the effect of the relative motion the two particles can have symmetrically the roles of impinging particle and target. Let $\theta$ be the 
angle between the directions of the dipole moment $\boldsymbol{P}_{\Gamma}$ of the DEMS formed by the direct interaction and the momentum $\boldsymbol{P}$ of the trajectory of the impinging particle, according to the $\theta$ angle definition, if we place arbitrarily the observer in the frame $S_{2}$ coinciding with the target particle, no energy and momentum can be associated to this observer because he cannot interact with himself, so assuming for example $m_{1}$ and $\boldsymbol{\beta}_{1}$ respectively the observed mass and the dimensionless velocity of an impinging particle \#1, from the Equation (1) we get

$$
\left\{\begin{array}{l}
\boldsymbol{P}_{c} \equiv \boldsymbol{P}_{1} c=m_{1} \boldsymbol{\beta}_{1} c^{2} \\
E \equiv E_{1}
\end{array}\right.
$$

In the BT approach exist a correlation between the first interaction distance and the wavelength that will have the source. In fact, the instant when two particles begin to interact corresponds to the birth of the DEMS which starts to emit with null luminosity when the particles are at $3 / 2$ of the minimal effective distance $\lambda$ that will be reached at the end of the A-phase. Following BT, the minimal effective distance defines the value of the characteristic wavelength of emission of the DEMS and is correlated with the instant in which the DEMS achieves the maximum luminosity. Since the delay time between the effective and the real positions of the impinging particle along its trajectory is always within the time interval $0 \leq t_{\text {delay }}<T$, the ratio between the distance $R\left(t_{\text {delay }}\right)$ of the two interacting particles at time $t_{\text {delay }}$ and the minimal effective distance of interaction $\lambda$ is equal to:

$$
\rho=\frac{R\left(t_{\text {delay }}\right)}{\lambda}=1+\beta \cos \theta,
$$

which value is in the interval $1 \leq \rho<2$. By using the Equation (8) and (9) we obtain the identity

$$
P c \cos \theta=(\rho-1) m_{1} c^{2}
$$

from which using Equation (8) and (10), the Equation (4) for the QRP becomes

$$
2 E_{\Gamma}\left[E_{1}-(\rho-1) m_{1} c^{2}\right]-\left[E_{1}^{2}-m_{1}^{2} \beta^{2} c^{4}\right]=0
$$

Considering a head-to-head collision at very high energy with $\theta \cong 0$ and $\beta \cong 1$, the Equation (9) converges to $\rho=2$ and the DEMS is created with the maximum available energy $E_{\Gamma} \equiv E_{1}$ coinciding with that one of the impinging particle measured by the observer placed in the frame of the target \#2. In these conditions the Equation (11) has solution

$$
E_{\Gamma} \equiv E_{1}=m_{1} c^{2}
$$

i.e. when the interaction occurs at very high velocity the energy of the DEMS converges to all the available relativistic energy of the source, equivalent to the total energy of the impinging particle \#1.

For interactions with energies involved lower than the maximum value of the 
available energy (12), the energy (6) is depending by the energy and momentum of the interacting particles.

In order to define a characteristic invariant term characterising the available energy for the DEMS, we use the numerator of the Equation (6).

Let

$$
\varepsilon_{1}^{2}=E_{1}^{2}-P_{1}^{2} c^{2}
$$

be a non-null non-impulsive squared term associated to the energy of the impinging particle \#1, using the momentum of the Equation (8) and the solution (12), we define

$$
E=\frac{\varepsilon_{1}}{\sqrt{1-\beta_{1}^{2}}}=\gamma_{1} \varepsilon_{1}
$$

where $\varepsilon_{1}$ is the rest energy involved in the DEMS production, measured in the c.m. of the interacting particle \#1 and $E_{1}$ is the total energy of the DEMS equivalent to that of the particle \#1 measured by the point of view of the observer placed on a the target particle \#2. Equation (14) is in agreement with the Special Relativity. Using the Equation (8) and (14), the energy and momentum of the DEMS produced or equivalently the energy and momentum of the impinging particle became

$$
\left\{\begin{array}{l}
\boldsymbol{P}=\boldsymbol{\beta}_{1} \gamma_{1} \frac{\varepsilon_{1}}{c} \\
E=\gamma_{1} \varepsilon_{1}
\end{array}\right.
$$

Since the Equation (6) represent the electromagnetic energy of the DEMS produced during the interaction between the impinging particle and the observer, the energy and momentum (15) are coinciding with the electromagnetic energy and momentum of a photon exchanged (statement (III)) between the two particles, which energy is characterised by a Doppler frequency due to the relative motion between source and observer.

The Equation (12) proves that the energy $E_{\Gamma}$ of the DEMS converges on the total electromagnetic energy $E$ of the interacting particles showing that the production of the DEMS is a physical process able to transform the rest energy $\varepsilon$ of a particle in the electromagnetic energy of the DEMS. The result (15) is the proof that the DEMS process produces a typical relativistic result starting from a typical quantum result. Since the total energy (14) of the impinging particle resulting from (13) can be positive or negative depending on whether we consider a particle or an antiparticle. The values of total energy inside the interval $-\varepsilon_{1}<E<\varepsilon_{1}$ are forbidden because an excitation of the particle with an energy greater than $2 \varepsilon_{1}$ may excite a negative energy particle up into the positive energy states $[6]$.

\section{Wave-Matter Duality: Compton and de Broglie Waves}

Many authors have conjectured that the de Broglie wave describing a particle is a 
real wave modulated by relativistic effects [7]. In agreement with the statement (III), using the Equations (6) and (15) to rewrite the electromagnetic energy and momentum exchanged between particle and observer

$$
\left\{\begin{array}{l}
E_{\Gamma}=\frac{\varepsilon}{2} \gamma \frac{1-\beta^{2}}{1-\beta \cos \theta} \\
P_{\Gamma}=\frac{\varepsilon}{2} \frac{\gamma \beta}{c} \frac{\frac{1}{\beta^{2}}-1}{\frac{1}{\beta}-\cos \theta}
\end{array}\right.
$$

the first of the Equation (16) can be interpreted as a relativistic Doppler effect due to the relative motion of the source emitting from its c.m. an energy $\varepsilon / 2$ along the sight line of the observer [8]. The Equation (16) describes energy and momentum of the impinging particle as a real electromagnetic wave source, emitting along the specific sight line on which is placed the observer on the target. For symmetry, we can exchange the roles of impinging particle and observer. During their reciprocal interaction if the angle of interaction is inside the interval $0<\theta \leq \pi$, and the particle moves with $\beta \ll 1$, frequency and wavelength of the exchange photon converge to the Compton values for an energy $\varepsilon / 2$ that can be considered the non-relativistic approximation:

$$
\left\{\begin{array}{l}
v_{c}=\varepsilon / 2 h \\
\lambda_{c}=2 h c / \varepsilon
\end{array}\right.
$$

on the otherwise for a very high energy collision, the angle of interaction during the interaction could be considered close to zero in such a way that frequency and wavelength converge to the de Broglie values for the relativistic energy $\gamma \varepsilon$

$$
\left\{\begin{array}{l}
v_{d b}=\gamma \varepsilon / h \\
\lambda_{d b}=h c / \beta \gamma \varepsilon
\end{array}\right.
$$

By considering the observer interacting with an impinging particle \#1, let $\varepsilon_{1}=m_{1} c^{2} / \gamma_{1}$ be the rest energy of the particle converted in electromagnetic energy and momentum (16), the correspondence between electromagnetic and mechanical values allows to consider a massive particle as an wave associated to de Broglie frequency and wavelength

$$
\left\{\begin{array}{l}
v_{d b}=\frac{\gamma_{1} \varepsilon_{1}}{h}=\frac{m_{1} c^{2}}{h} \\
\lambda_{d b}=\frac{h c}{\beta_{1} \gamma_{1} \varepsilon_{1}}=\frac{h}{\beta_{1} m_{1} c}
\end{array}\right.
$$

in general, the Compton wave of a particle is a de Broglie wave in non-relativistic conditions.

\subsection{Observation of an Interacting Pair in the Lab}

From the symmetry of the observers placed in the frames $S_{1}$ and $S_{2}$, each of them measures reciprocally the energy of the respective impinging particle without 
being able to measure their own rest energy. In fact, in order to make a measure of energy in these two frames, they need to create a local DEMS, but in each one of the frames $S_{1}$ and $S_{2}$ just a single charge is placed, so the observers cannot perform local measurements of their energy and momentum.

By defining a lab-frame in which a polarisable neutral medium as ordinary matter or vacuum can interact, when an interaction between a pair of charges coinciding with the frames $\# 1$ and $\# 2$ occurs, the neutral medium reacts with the particles producing two independent DEMS: $S S_{1}, S S_{2}$.

From the experimental point of view, in order to observe the collision of the two particles we cannot use directly the frames $S_{1}$ and $S_{2}$, because the DEMS $S_{1} S_{2}$ and the symmetrical one, do not allow simultaneous measurements of energy and momentum of the two colliding particles. Instead, using the lab $S$ we can measure energy and momentum of the c.m. of all the subjects involved in the collision. In fact, the lab is sensible to the total energy involved, so applying the Equation (16) to the interaction between the lab at rest and each of the two particles \#1 and \#2, we get for each particle involved in the interaction:

$$
S S_{j}\left\{\begin{array}{l}
E_{j}=\gamma_{j} \varepsilon_{j} \\
\boldsymbol{P}_{j}=\gamma_{j} \boldsymbol{\beta}_{j} \frac{\varepsilon_{j}}{c}
\end{array}\right.
$$

where $\varepsilon_{j}$ is the rest energy of each particle involved. The Lorentz factor and the relative velocity of the particles in Equation (20) are both referred to the same lab.

\subsection{Observation of a DEMS in the Lab}

To calculate in the lab $S$ the total energy and momentum of the c.m. $S_{0}$ of the DEMS produced by the two interacting particles, we must obtain the explicit expressions of the total rest energy and of the factors $\beta$ and $\gamma$. By using the invariant (13) and the Equation (1) and (20) for the two involved particles with rest energy and dimensionless velocity $\left(\varepsilon_{i}, \boldsymbol{\beta}_{i}\right)$, is obtained the total rest energy:

$$
\varepsilon=\sqrt{\left(E_{1}+E_{2}\right)^{2}-\left\|\boldsymbol{P}_{1}+\boldsymbol{P}_{2}\right\|^{2} c^{2}}=\sqrt{\varepsilon_{1}^{2}+\varepsilon_{2}^{2}+2 \varepsilon_{1} \varepsilon_{2} \gamma_{1} \gamma_{2}\left(1-\beta_{1} \beta_{2} \cos \delta\right)}
$$

where $\delta$ is the angle between the momenta $\boldsymbol{P}_{1}$ and $\boldsymbol{P}_{2}$ of the two colliding particles with respect to the lab-frame $S$.

Since the c.m. $S_{0}$ moves with respect $S$ with a velocity $\beta$

$$
\boldsymbol{\beta}=\frac{\left(\boldsymbol{P}_{1}+\boldsymbol{P}_{2}\right) c}{E_{1}+E_{2}}=\frac{\varepsilon_{1} \gamma_{1} \boldsymbol{\beta}_{1}+\varepsilon_{2} \gamma_{2} \boldsymbol{\beta}_{2}}{\varepsilon_{1} \gamma_{1}+\varepsilon_{2} \gamma_{2}} .
$$

To obtain $\gamma$, by using the total rest energy (21) we get:

$$
\gamma=\frac{E_{1}+E_{2}}{\varepsilon}=\frac{\varepsilon_{1} \gamma_{1}+\varepsilon_{2} \gamma_{2}}{\varepsilon} .
$$

Using now the Equation (21) and (22) with the (23), energy and momentum of the c.m. of the DEMS produced during the interaction of the two particles with respect to the lab-frame, in agreement with the Equation (1) and (15) is 
given by

$$
S S_{0}\left\{\begin{array}{l}
E=\gamma \varepsilon \equiv \varepsilon_{1} \gamma_{1}+\varepsilon_{2} \gamma_{2} \\
\boldsymbol{P}=\gamma \boldsymbol{\beta} \frac{\varepsilon}{c} \equiv \frac{\varepsilon_{1}}{c} \gamma_{1} \boldsymbol{\beta}_{1}+\frac{\varepsilon_{2}}{c} \gamma_{2} \boldsymbol{\beta}_{2} .
\end{array}\right.
$$

In this case $S S_{0}$ is a connection between lab-frame and c.m. of the DEMS $S_{1} S_{2}$, Equation (24) is equivalent to a pair of DEMS $S S_{1}$ and $S S_{2}$.

\subsection{Doppler Effect in the Lab-Frame}

The dynamical state of one interacting particle respect an observer is defined by the energy and momentum of the DEMS produced. Since the frame $S_{0}$ associated to the c.m. of a DEMS is in motion with respect each observer in the universe, using the energy of the Equation (16) we define a characteristic wavelength $\lambda_{0}$ connecting the c.m. of the DEMS with the observer. The wave at low energy corresponds to the Compton wavelength and at high energy is modulated by a Doppler factor due to the relative motion between the two frames $S$ and $S_{0}$ :

$$
\lambda_{0}=\lambda_{c} \gamma(1-\beta \cos \theta),
$$

the wavelength of the c.m. (25) is a generalization of the Compton wavelength of the source for observers in relative motion respect the c.m. of the DEMS, proving as the impinging particle has a wave behaviour respect the observer [9] when the observer interact trough out the wave emitted by a DEMS produced in the lab, i.e. interacts indirectly, but has a particle behaviour as described in the Equation (20) when they interacts directly with the observer producing a DEMS.

\section{Application to the Compton Experiment Phenomenology}

A historical step in the birth of the Quantum Mechanics was done with the Compton experiment. In this paragraph is explained the Compton's experiment without to use the usual approach.

When a pair electron-positron interact with respect to the lab frame $S$ at very low relative velocity $\beta_{1} \approx \beta_{2} \approx 0$ it follows $\beta \approx 0$ and $\gamma \approx 1$, Equation (21) gives a rest energy $\varepsilon \approx 2 m_{e} c^{2}$ coinciding with the total energy of the particles, i.e. the Compton and the de Broglie wavelengths have an identical value. Under these conditions the wavelength (25) of a photon emitted from the c.m. $S_{0}$ of the DEMS toward an atomic electron is the Compton's wavelength (17) of the incoming electron or positron in which the carried rest energy is all that of the pair.

Now following the experimental procedure, we know that during a collision of an X-ray photon with an orbital electron of an atom of graphite, the photon could be bounced away only like massive particles can do. Following BT phenomenology, when the photon with energy $E$ and momentum $P$ impinges on an electron at rest, the intense electric field existing in the neighbourhood of the atomic electron polarises locally the photon in a electron-positron virtual pair with a near zero kinetic energy. The pair can live only a short time during which 
the atomic electron interacting with the virtual positron of the pair produces a new real DEMS which is observed in the lab-frame as a bounced photon with energy and momentum $E^{\prime}<E$ and $P^{\prime}<P$. The residual virtual electron of the pair not directly involved in the DEMS production is scattered away with an amount of energy and momentum bigger than the energy of the atomic electron. The phenomenology above, describes a process in which two electrons exchange their role. In fact, the atomic electron and the virtual electron of the pair switch their physical identities.

The wavelength of the photon bounced away and collected in the lab-frame $S$ along a direction forming an angle $\theta$ with the beam axis, is different from the wavelength produced in the DEMS performed by the positron-electron interaction during the $\mathrm{X}$-electron scattering. In fact, the virtual positron interacting with the atomic electron of the graphite produces a low energy local DEMS in the layer, with a resulting wavelength equal to the Compton one, whereas in the lab-frame $S$, on the calorimeter are collected all the photons emitted by the DEMS with a de Broglie wavelength. Hence considering the X-electron scattering, when the polarised positrons interact with the atomic electrons the energy of the interaction is low enough to converge in the c.m. of the DEMS to the Compton wavelength of the positron. By using this local equivalence, we combine the Compton and de Broglie wavelengths and frequencies obtaining in agreement with the formal result proposed by de la Peña and Cetto and Kracklauer the transformation

$$
\left\{\begin{array}{l}
\lambda_{d b}=\frac{\lambda_{c}}{\beta \gamma} \\
v_{d b}=\gamma v_{c}
\end{array}\right.
$$

from the Compton wavelength and frequency in the frame of the c. m. to the de Broglie wavelength and frequency in the lab-frame [10] [11].

To analyse the emission of the DEMS produced in the graphite layer, i.e. the Doppler modulations (25) of the Compton wavelength $\lambda_{c}$ of the scattered photons varying with respect to the collection angle $\theta$, we use the $S_{0}$ frame of the c.m. of the DEMS produced during the positron-electron interaction. Since velocity and Compton wavelength are constant with respect to the variation of the angle, the differential of the Doppler wavelength (25) gives

$$
\mathrm{d} \lambda_{0}=\beta \gamma \lambda_{c} \sin \theta \mathrm{d} \theta
$$

which describes in the frame $S_{0}$ of the scattered photon the differential behaviour of the generalised Compton wavelength of the DEMS. Applying the transformation (26) to the Equation (27) in order to obtain the de Broglie wavelength variation in the frame $S$ where the photons are collected

$$
\mathrm{d} \lambda=\lambda_{c} \sin \theta \mathrm{d} \theta
$$

integrating on an arbitrary interval $\left[\lambda, \lambda^{\prime}\right]$ corresponding to an angular interval $[0, \theta]$ in which are collected the scattered photons. Using the Compton wave- 
length definition (17) where the rest energy (21) in terms of rest mass gives $\varepsilon=2 m_{e} c^{2}$ Equation (28) becomes:

$$
\lambda^{\prime}-\lambda=\frac{h}{m_{e} c}(1-\cos \theta)
$$

fully in agreement with the experimental result.

\section{Electron-Positron Annihilation}

If we consider the lab-frame placed in the c.m. of the two impinging particles, in such a way that the observed energy along the sight line is half of the total energy (16) of the DEMS

$$
E_{\Gamma}=\frac{\gamma \varepsilon}{2}
$$

the angle at which the DEMS is able to emit two photons with an energy equal to half of the total energy of the source produced is:

$$
\theta=\operatorname{arcos} \sqrt{1-\frac{1}{\gamma^{2}}} .
$$

Considering a low energy $\gamma \cong 1$, the electron-positron annihilation occurs with a null total momentum and a total energy $\varepsilon \cong 2 m_{e} c^{2}$.

In agreement with the Equation (31), we observe in the lab-frame the emission of two opposite photons at an angle of $90^{\circ}$ respect the trajectory of the two colliding particles, each with a frequency coinciding with the Compton one $v \cong \frac{m_{e} c^{2}}{h}$.

In general, for $\gamma>1$ the c.m. of the pair is moving away from the lab-frame with a speed $\beta c$ and the photon emission occurs with two symmetric angles of scattering lower than $90^{\circ}$ degrees.

Using the principle of the time reversal we can consider a pair creation from a polarized source. In this case at low energy electrons can be created and successively annihilated producing two photons.

\section{The Cherenkov Limit Angle}

In order to define the emission angle in which a particle crossing a media $S$ is seen to emit photons by an observer, according to the existence and positivity of the Doppler energy (16) we have:

$$
\cos \theta<\frac{1}{\beta}
$$

always verified in vacuum where the source can emit photons in any direction, whereas for observers embedded in a polarizable medium with a relative refraction index $n>1$ Equation (32) gives for a crossing particle:

$$
\theta \geq \operatorname{arcos} \frac{1}{n \beta}
$$


describing the characteristic Cherenkov effect with a limit angle for the photon emission in a medium crossed by electrical particles moving at velocity greater then light in the medium.

\section{Deduction of the Lorentz-Einstein's Transformations}

The use of the generalised Compton wavelength allows to deduce the Lorentz-Einstein transformations, in fact, if a particle interacts in the lab-frame with many antiparticles each DEMS produced emits a wave. When the waves achieve the positions of two different observers, each wave allows to the observers to perform independent measures of energy and momentum of the same interacting particle, each wave is associated to a measure of time and position of the same particle. Is fundamental to know how the measures are interconnected.

We consider the c.m. $S_{0}$ of a DEMS placed in a whatever lab in space. When two different observers receive the electromagnetic signal emitted by the same DEMS $S_{0}$, the Compton wave is observed in two different places $S$ and S' with different wavelength for the Doppler effect (25) produced between the DEMS and each observer.

Assuming arbitrarily the observer $S$ to be at rest with respect $S_{0}$ in such a way that the emission of the DEMS along the sight line connecting $S_{0}$ to $S$ forms a null angle with respect the direction of observation, and the observer $S$ to be in motion in such a way that the sight line connecting $S_{0}$ to $S$ forms a non null angle with respect to the direction of emission connecting $S_{0}$ and $S$, the measures of time and position of the event "emission from the c.m. $S_{0}$ of the DEMS", realized independently by the two observers $S$ and $S$ are connected by the generalized Compton wavelengths (25):

$$
\frac{x}{\lambda_{c}}=\frac{c t}{\lambda_{c}}=\frac{x^{\prime}}{\lambda_{0}}=\frac{c t^{\prime}}{\lambda_{0}}=a
$$

where $a>0$ is equal for each observer. Using the Equation (25) and (34), we write two space and time transformations able to interconnect the measures of position and time of the two inertial observers $S$ and $S$ :

$$
\left\{\begin{array}{l}
x^{\prime}=\gamma(x-v t \cos \theta) \\
t^{\prime}=\gamma\left(t-v \frac{x}{c^{2}} \cos \theta\right)
\end{array}\right.
$$

the transformations (35) agree with the generalized Lorentz-Einstein's transformations, which in standard configuration $(\theta=0)$ describe the observer $\mathcal{S}$ moving along the $X$-axis toward the observer $S$.

In this context the derivation of the relativistic transformations (35) takes origin from a typical EM wave-connection between the DEMS and the observers. This result proves that the measures of space and time can be performed only if the two observers $S$ and $S$ are reached by the same wave signal emitted by a DEMS in $S_{0}$. To do that is necessary each pair of observers are causally connected with a same DEMS. In general if a particle moving in spacetime produc- 
ing a great number of DEMS, each in a different points $P$ of space, each observer can be connected with all the points $P$ of space by means of the own interaction with all the DEMS produced, i.e. the wavelengths of each DEMS realise a different metric (34) for each pair of observers and empty spacetime is not a static primitive geometric notion, but a variable active consequence of the existence of infinite wave connections among observers.

\section{Information Transfer between Two or More Observers}

In Relativity a phenomenon of great relevance and mystery is the impossibility to define an inertial frame $S$ with respect of which a system $S_{0}$ in motion with respect to the frame $S$ has a relative velocity greater than $c$. Analysing this effect in the present context, considering a signal as an elementary information, is proved as the direct transferring of information violates the cause-effect principle, because occurs with a superluminal velocity. On the otherwise, when the transferring of information is produced indirectly by successive interactions involving two or more inertial observers, the velocity cannot exceed the speed of light.

To prove that is considered the differentials of the Equation (34) for a motion between the observers $S$ and $S$ at speed component $\pm v_{x}=v \cos \theta$ :

$$
\begin{aligned}
& \mathrm{d} x^{\prime}=\gamma\left(\mathrm{d} x \pm v_{x} \mathrm{~d} t\right) \\
& \mathrm{d} t^{\prime}=\gamma\left(\mathrm{d} t \pm v_{x} \frac{\mathrm{d} x}{c^{2}}\right)
\end{aligned}
$$

Dividing the differential of space with the one of the time, Equations (36) give the usual relativistic sum of the velocity for an observer $S_{0}$ in motion with speed component $u_{x}$ along the $X$-axis in the frame $S$ in relative motion with speed component $v_{x}$ respect $S$

$$
u_{x}^{\prime}=\frac{\mathrm{d} x^{\prime}}{\mathrm{d} t^{\prime}}=\frac{u_{x} \pm v_{x}}{1 \pm \frac{u_{x} v_{x}}{c^{2}}}
$$

If the motion of $S_{0}$ with speed $u_{x}$ respect to $S$ or the motion of $S$ with speed $v_{x}$ respect to $S$ were to occur at infinite velocity, the relativistic sum of the velocities (37) cannot be infinite but would give respectively $c^{2} / v_{x}$ or $c^{2} / u_{x}$, each value of which is a finite superluminal speed coinciding with the phase velocity of the de Broglie's wave observed in the frame $S$ as it were emitted respectively from a DEMS associated to the moving frames $S$ and $S_{0}$.

\subsection{Superluminal Communication between Two Interacting Observers}

In order to the previous consideration we take in to account a pair of charged particles placed in the frames $S_{1}$ and $S_{2}$. When two charged particles reach a reciprocal distance of interaction equal to $3 / 2$ of the wavelength of the source zone of the DEMS that will produced, the principle of cause-effect was been violated. In that instant the source zone starts to expand it localising inside the spherical crown of the source zone centred in the c.m. of the interacting pair, an amount 
of energy and momentum greater than zero. The energy localised inside the source zone increases until the charges reach the minimum interaction distance compatible with their trajectories, this minimum value sets the final wavelength $\lambda$ of the DEMS equivalent to the exchanged photon. How does the source before that the phase A of the interaction ends, at know the minimum distance of interaction? During the DEMS formation, the progressive expansion of an ideal spherical surface from the initial diameter $\lambda$ and the final diameter $3 \lambda / 2$ delimits the source zone. During the expansion, the diameter of the surface increases with the angular phase $\varphi$ of the spinning of the field of the Poynting vector around the DEMS. Its expansion speed is $u_{\exp }=c / \varphi$, i.e. when at the beginning the phase is $\varphi=0$ the source zone starts to expand it with an infinite velocity decreasing rapidly with the phase value. After a time $T / 2$ the source zone ends to expand it and the DEMS starts to emit as an ideal electromagnetic dipole.

Considering the observer $S_{1}$ placed on the particle \#1 in motion with a relative velocity $v_{12}$, at $\varphi=0$ using Equation (37) the observer $S_{2}$ measures the velocity of propagation of the spherical front of the DEMS as

$$
u_{\text {exp }}^{\prime}=\lim _{\varphi \rightarrow 0} \frac{\frac{c}{\varphi}+v_{x}}{1+\frac{v_{x}}{\varphi c}}=\frac{c^{2}}{v_{x}}
$$

coinciding with the speed of the de Broglie wave (19). In this sense the de Broglie wave carries superluminally the information of the reciprocal interaction in a way such that each particle know the energy and momentum that will be exchanged before really the true interaction occurs.

\subsection{Subluminal Communication between Inertial Observers}

Considering the relative velocity $\boldsymbol{u}=\left(u_{x}, 0,0\right)$ of an observer placed in $S_{0}$ with respect an observer $S$, the transformations (38) give the velocity of $S_{0}$ with respect the observer $\mathcal{S}$ when $S$ and $S$ are in relative motion along the $X$-axis at a velocity $\boldsymbol{v}=(v \cos \theta, 0,0)$.

Since the position of $S_{0}$ on the $X$-axis is settled by the coordinate $x=a \lambda_{0}$ on the lab-frame $S$ at time $t=a T_{0}$, where wavelength and period characterise the wave signal emitted towards the observer $S$ from the DEMS placed in $S_{0}$. The Compton's wave emitted from $S_{0}$ is received in the frame $S$ in a Doppler wave mode coinciding with a de Broglie's wave, so to measure position and time of $S_{0}$ with respect $S$ we apply the transformations (26) on the signal emitted from $S_{0}$ obtaining:

$$
\left\{\begin{array}{l}
x=a \frac{\lambda_{0}}{\beta_{x} \gamma} \\
t=a \frac{\lambda_{0}}{\gamma c}
\end{array}\right.
$$

Since $S_{0}$ is in relative motion with respect the frame $S$ at velocity $u_{x}$, calcu- 
lating the speed component $u_{x}^{*}=\mathrm{d} x / \mathrm{d} t$ of the DEMS in $S_{0}$ with respect the observer $S$, the Equation (39) gives

$$
u_{x}^{*}=\frac{c}{\beta_{x}} \equiv \frac{c^{2}}{u_{x}}
$$

which is equal to the phase velocity of the de Broglie's wave received in the frame $S$.

Considering a particle \#1 in relative motion respect the observer in the lab-frame $S$, the direct interaction produces a DEMS $S S_{1}$ connecting the two systems with an electromagnetic signal propagating with superluminal velocity (40). Similarly occurs considering the direct interaction between the frames $S$ and $S$, the direct interaction connects the observers with a signal propagating with velocity

$$
v_{x}^{*}=\frac{c}{\beta_{x}^{\prime}} \equiv \frac{c^{2}}{v \cos \theta}
$$

To obtain information about the velocity of a system $S_{0}$ respect the frame $S$ we use the Equation (39) obtaining

$$
u_{x}^{\prime}=\frac{u_{x}^{*}+v_{x}^{*}}{1+\frac{u_{x}^{*} v_{x}^{*}}{c^{2}}}=\frac{u_{x}+v \cos \theta}{1+\frac{u_{x} v \cos \theta}{c^{2}}}
$$

always lower than $c$. The Equation (40) and (42) prove that the observer $S$ can measure for an incoming particle a velocity greater than $c$ only when interacts directly with it, in this case the wave emitted toward $S$ is a monochromatic Compton's wave, received as a shifted Doppler wave due to the relative motion of the impinging particle in the frame. The wave measured by the observer is coinciding with the de Broglie's wave of the impinging particle. For observations occurring in a frame $S$ on which are collected in a unique wave-packet more waves, each emitted from a DEMS produced during the direct interaction of the particle with a different inertial observer, the velocity measured by the Equation (40) is equivalent at the group velocity of the wave-packet describing the particle, always lower than the light speed.

\subsection{Direct Entanglement}

By considering the case in which two particles are created in pair with a kinetic energy enough to escape each from the other, this is the act of formation of a primary DEMS in which the two particles vary their interaction distance with continuity increasing the wavelength due to the direct interaction and remaining entangled for ever with their direct connection. In fact, the DEMS has a decreasing wavelength in time but the Planck's action of the corresponding exchanged photons remains with the same value. Each interaction involving one of the two particles in time, for the energy and momentum conservation laws of the DEMS, maintain the correlation in spacetime in such a way that any modification of spin, energy and momentum occurring on one of the two particles, produces a simultaneous correlate modification in the twin particle, independently 
from their effective distance. The modification is occurring at superluminal velocity (40) and corresponds to an instantaneous revision of the direct interaction to maintain continuously unchanged the electromagnetic structure of the DEMS which for a free direct interaction has a constant coupling value $\alpha$.

\section{Conclusion}

In order to reduce the number of models and theories required to describe exhaustively a phenomenon with manifold aspects, are ever necessary new powerful ideas. In this article an extension of the Bridge Theory is proposed to describe in a single self-consistent theoretical and phenomenological context the quantum-relativistic behaviours appearing experimentally in the interactions among photons and charged particles. The present theoretical formulation proves that the wave-particle duality is the experimental evidence of a common origin of two much different behaviours of a charged particle, as the ones described by Quantum Mechanics and by Special Relativity. The emerging theory shows that superluminality and entanglement both have to do with the direct electromagnetic interaction of a pair of charged particles producing a DEMS. In fact, after the production of the dipole, the electromagnetic source continues to exist independently by their effective distance achieved during their removal phase and independently by the occasional external actions that could occur on one of the two particles forming the DEMS. Each action modifying the dynamic state of one of the two particles produces an instantaneous reaction on the dynamic state of the other to keep their coupling constant. In fact, for all free interactions, independently of the dynamics of the two particles and their distances, the constant of fine structure is numerically invariant.

\section{Acknowledgements}

With great pleasure I thank Prof. Guido Dematteis for his contribution to the understanding of this new emerging theory.

\section{Conflicts of Interest}

The authors declare no conflicts of interest regarding the publication of this paper.

\section{References}

[1] Auci, M. and Dematteis, G. (1999) International Journal of Modern Physics, B13, 1525-1557. https://doi.org/10.1142/S0217979299001569

[2] Auci, M. (1989) Physics Letters A, 135, 86-88. https://doi.org/10.1016/0375-9601(89)90650-6

[3] Auci, M. (2003) On the Compatibility between Quantum and Relativistic Effects in an Electromagnetic Bridge Theory. arXiv:1003.3861

[4] Auci, M. (1990) Physics Letters A, 148, 399-404. https://doi.org/10.1016/0375-9601(90)90488-A 
[5] Auci, M. (1990) Physics Letters A, 150, 143-150. https://doi.org/10.1016/0375-9601(90)90109-2

[6] Feshback, H. and Villars, F. (1958) Reviews of Modern Physics, 30, 24. https://doi.org/10.1103/RevModPhys.30.24

[7] Bhaumik, M. (2016) Deciphering the Enigma of Wave-Partcle Duality. arXiv:1611.00226

[8] Einstein, A. (1905) Annalen der Physik, 17, 891. https://doi.org/10.1002/andp.19053221004

[9] Auci, M. (2003) Wave-Particle Behaviour in Bridge Theory. arXiv:1201.4577

[10] de la Peña, L. and Cetto, M. (1996) The Quantum Dice: An Introduction to Stochastic Electrodynamics. Kluwer Academic Publishers, Norwell, MA, Chap. 12. https://doi.org/10.1007/978-94-015-8723-5

[11] Kracklauer, A.F. (1992) Physics Essays, 5, 226. https://doi.org/10.4006/1.3028975 